\title{
Discovery and validation of an expression signature for recurrence prediction in high-risk diffuse-type gastric cancer
}

\author{
In-Seob Lee ${ }^{1,2} \cdot$ Divya Sahu $^{1} \cdot$ Hoon Hur ${ }^{3,4} \cdot$ Jeong-Hwan Yook ${ }^{2} \cdot$ Byung-Sik Kim $^{2} \cdot$ Ajay Goel $^{1}$ (D)
}

Received: 2 November 2020 / Accepted: 29 December 2020 / Published online: 1 February 2021

(C) The International Gastric Cancer Association and The Japanese Gastric Cancer Association 2021

\begin{abstract}
Background Diffuse type gastric cancer (DGC), represented by low sensitivity to chemotherapy and poor prognosis, is a heterogenous malignancy in which patient subsets exhibit diverse oncological risk-profiles. This study aimed to develop molecular biomarkers for robust prognostic risk-stratification and improve survival outcomes in patients with diffuse type gastric cancer (DGC).

Methods We undertook a systematic and comprehensive discovery and validation effort to identify recurrence prediction biomarkers by analyzing genome-wide transcriptomic profiling data from 157 patients with DGC, followed by their validation in 254 patients from 2 clinical cohorts.

Results Genome-wide transcriptomic profiling identified a 7-gene panel for robust prediction of recurrence in DGC patients $(\mathrm{AUC}=0.91)$, which was successfully validated in an independent dataset $(\mathrm{AUC}=0.86)$. Examination of 180 specimens from a training cohort allowed us to establish a gene-based risk prediction model ( $\mathrm{AUC}=0.78 ; 95 \% \mathrm{CI} 0.71-0.84$ ), which was subsequently validated in an independent cohort of $74 \mathrm{GC}$ patients (AUC $=0.83 ; 95 \% \mathrm{CI} 0.72-0.90$ ). The Kaplan-Meier analyses exhibited a consistently superior performance of our risk-prediction model in the identification of high- and lowrisk patient subgroups, which was significantly improved when we combined our gene signature with the tumor stage in both clinical cohorts (AUC of 0.83 in the training cohort and 0.89 in the validation cohort). Finally, for an easier clinical translation, we established a nomogram that robustly predicted prognosis in patients with DGC.

Conclusions Our novel transcriptomic signature for risk-stratification and identification of high-risk patients with recurrence could serve as an important clinical decision-making tool in patients with DGC.
\end{abstract}

Keywords Gastric cancer · Diffuse type $\cdot$ Recurrence $\cdot$ Biomarker $\cdot$ Transcriptomic

\section{Introduction}

Supplementary Information The online version contains supplementary material available at https://doi.org/10.1007/s1012 0-021-01155-y.

Ajay Goel

ajgoel@coh.org

1 Department of Molecular Diagnostics and Experimental Therapeutics, Beckman Research Institute of City of Hope Comprehensive Cancer Center, Biomedical Research Center, 1218 S. Fifth Avenue, Monrovia, CA 91016, USA

2 Department of Surgery, Asan Medical Center, University of Ulsan College of Medicine, Seoul, South Korea

3 Department of Surgery, Ajou University of School of Medicine, Suwon, South Korea

4 Cancer Biology Graduate Program, Ajou University Graduate School of Medicine, Suwon, South Korea
Gastric cancer (GC) is one of the most frequently diagnosed malignancies and the second leading cause of cancer-related deaths worldwide [1]. The majority of GC presents at an advanced stage, and consequently, a significant number of patients experience recurrence following surgery. Although introduction of adjuvant or perioperative chemotherapy has reduced tumor recurrence rates and improved survival in patients with resectable GC, the overall survival benefit from these interventions is only $\sim 10 \%$ and the prognosis of a majority of patients still remains unsatisfactory [2-4]. This clinical challenge is further compounded due to the inherent heterogeneity in response to various treatment approaches even within the same disease stage, as some patients can be effectively cured through surgery alone, while others do not benefit when offered adjuvant chemotherapy following 
surgical removal of their primary lesion. This conundrum has been highlighted in some of the recent studies that elegantly demonstrate that the prognosis and chemotherapeutic response in patients is largely dependent not on the disease stage but is heavily dictated by the underlying tumor subtype $[5,6]$. These data are of tremendous value as these emphasize that the currently used risk stratification criteria in the clinic that predominantly rely on the TNM staging are inadequate in predicting the prognosis of GC patients, and hence, unable to guide the optimal treatment plan in them.

Diffuse type gastric cancer (DGC), one of two major histologic subtypes of GC as per Lauren's classification is characterized by low sensitivity to 5-fluorouracil based chemotherapy and its association with poor prognosis $[7,8]$. In the Cancer Genome Atlas (TCGA) analysis, genomically stable (GS) tumors are represented by enrichment of diffuse type histology and mutations in the $\mathrm{CDHI}$ and $\mathrm{RHOA}$ genes, which exclusively characterize DGC [9]. In addition, a study from the Asian Cancer Research Group (ACRG) classifying tumors based on the presence of microsatellite instability (MSI), epithelial-mesenchymal transition (EMT), and TP53 mutations revealed that mesenchymal-like (MSS/EMT) type frequently corresponds to DGC [10]. Clinical analyses based on molecular classifications consistently ascertained that phenotypes with diffuse histology are associated with poor prognosis among subtypes in GC $[10,11]$. Although aggressive tumor behavior and suboptimal therapeutic options are primarily responsible for the poor outcomes in patients with DGC, the lack of clinically robust biomarkers that can allow identification of patients at a higher risk for disease recurrence and determine the likelihood of chemotherapy benefit are much needed. Collectively, this highlights the importance for developing clinically relevant biomarkers for riskstratification in patients with DGC.

Recent studies have somewhat improved our current understanding of DGC based on genomic analysis and have allowed its categorization into two subtypes-one with infrequent genetic changes and low clonality, and the other with higher mutational burden and clonality [12]. These findings suggest that tumors with diffuse histology can be further classified into various subtypes, which has led to growing endeavors to identify specific biomarkers that can inform prognostic implications in everyday clinical practice. A few studies have analyzed clinical specimens from patients with DGC in order to identify tissue-derived transcriptomic and proteomic markers for risk-stratification [13, 14]. While findings from these studies have been encouraging, certain shortcomings of these efforts include the non-comprehensive biomarker discovery and validation process, and lack of independent validation of these biomarkers for their translation into clinical settings. Herein, we have addressed these limitations of the existing literature by performing a systematic and genome-wide transcriptomic expression profiling in tissue specimens from patients with DGC, followed by rigorous bioinformatic approaches to identify a panel of genes that could robustly predict recurrence following surgery and adjuvant treatment. Following the discovery of such a 7-gene panel, we established a risk-prediction model, which was successfully validated in two independent cohorts of patients with DGC. We finally developed a nomogram to assess the prognosis in this subset of patients.

\section{Materials and methods}

This study was performed in accordance with the REMARK (Reporting recommendations for tumor MARKer prognostic studies) guidelines [15].

\section{Biomarker discovery and validation using transcriptomic expression profiling data}

For the biomarker discovery phase, we analyzed genomewide expression profiling data from three publicly available datasets (GSE62254, GSE13861, and TCGA-STAD). Expression data and corresponding clinical information were downloaded from the Gene Expression Omnibus (GEO) database and UCSC Xena Browser (https://xenabrowse r.net). Three cohorts included a total of $845 \mathrm{GC}$ patients. The GSE62254 cohort included 300 GC cases; of which 114 cases were selected with a histology of poorly differentiated tubular adenocarcinoma or signet ring cell carcinoma as defined by the World Health Organization categorization and diffuse type by Lauren's classification [16]. After exclusion of 17 cases accompanied with distant metastasis and six patients lacking information on disease recurrence, 91 cases were included in the final analysis. In the GSE13861 dataset consisting of $65 \mathrm{GC}$ cases, there were 30 patients with diffuse type histology. Among them, four cases with distant metastasis were excluded and remaining 26 cases were analyzed. In the TCGA cohort including 81 diffuse type GC cases, 39 patients without recurrence information and 2 cases lacking data on distant metastasis were excluded. Finally, 157 cases were included in the analysis, and their clinicopathologic characteristics are summarized in Table S1.

\section{Clinical cohorts for biomarker validation}

A total of 254 patients with GC, from two independent cohorts, were enrolled for the training and validation of recurrence prediction biomarkers (Table 1). The training cohort comprised of patients enrolled at the University of Ulsan and Asan Medical Center, Seoul, Korea, and the validation cohort patients enrolled at the Ajou University, Suwon, Korea. All patients received curative surgery for 
Table 1 Clinicopathologic characteristics of the two clinical cohorts with diffuse type gastric cancer

\begin{tabular}{|c|c|c|c|}
\hline \multirow[t]{2}{*}{ Variables } & \multicolumn{2}{|l|}{ Tissue samples, $n(\%)$} & \multirow[t]{2}{*}{$P$-value } \\
\hline & Training cohort $(n=180)$ & Validation cohort $(n=74)$ & \\
\hline $\begin{array}{l}\text { Median age at opera- } \\
\text { tion, years (range) }\end{array}$ & $59(18-83)$ & $52(28-83)$ & 0.082 \\
\hline \multicolumn{4}{|l|}{ Age distribution } \\
\hline$\leq 50$ & $45(25.0)$ & $31(41.9)$ & \multirow[t]{2}{*}{0.008} \\
\hline$>50$ & $135(75.0)$ & $43(58.1)$ & \\
\hline \multicolumn{4}{|l|}{ Gender } \\
\hline Male & $112(62.2)$ & $45(60.8)$ & \multirow[t]{2}{*}{0.833} \\
\hline Female & $68(37.8)$ & $29(39.2)$ & \\
\hline \multicolumn{4}{|l|}{ Tumor location } \\
\hline Upper third & $43(23.9)$ & $19(25.7)$ & \multirow[t]{4}{*}{ NA } \\
\hline Middle third & $43(23.9)$ & $34(45.9)$ & \\
\hline Lower third & $80(44.4)$ & $18(24.3)$ & \\
\hline Entire & $14(7.8)$ & $3(4.1)$ & \\
\hline \multicolumn{4}{|l|}{ TNM stage* } \\
\hline 1 & $12(6.7)$ & $6(8.1)$ & \multirow[t]{3}{*}{0.783} \\
\hline 2 & $66(36.7)$ & $24(32.4)$ & \\
\hline 3 & $102(56.7)$ & $44(59.5)$ & \\
\hline \multicolumn{4}{|l|}{ Lymphovascular invasion } \\
\hline Present & $125(69.4)$ & $50(67.6)$ & \multirow[t]{2}{*}{0.769} \\
\hline Not identified & $55(30.6)$ & $24(32.4)$ & \\
\hline \multicolumn{4}{|l|}{ Adjuvant chemotherapy } \\
\hline Yes & $125(69.4)$ & $50(67.6)$ & \multirow[t]{2}{*}{0.234} \\
\hline No & $55(30.6)$ & $24(32.4)$ & \\
\hline \multicolumn{4}{|l|}{ Recurrence } \\
\hline Yes & $61(33.9)$ & $26(35.1)$ & \multirow[t]{2}{*}{0.849} \\
\hline No & $119(66.1)$ & $48(64.9)$ & \\
\hline $\begin{array}{l}\text { Median follow-up } \\
\text { period, months (range) }\end{array}$ & $48.9(3.2-96.4)$ & $43.2(8.2-90.5)$ & 0.169 \\
\hline
\end{tabular}

*TNM stage was based on AJCC 7th edition biopsy-proven primary DGC between 2008 and 2014. Patients who received neoadjuvant treatment and those in whom the cancers developed in the remnant stomach following previous partial gastrectomy were excluded. The tumor recurrence was assessed by a laboratory test, endoscopy, and abdominopelvic $\mathrm{CT}$ on a regular basis, as recommended by the gastric cancer treatment guidelines [17]. The tumor staging was determined according to the 7th edition of the American Joint Committee on Cancer [18]. Details of tissue preparation and pathologic interpretation are provided in Supplementary methods.

All procedures were conducted in accordance with the Declaration of Helsinki. A written informed consent was obtained from all participants. This study was approved by the Institutional Review Board of Asan Medical Center (Study No. 2019-0237) and Ajou university hospital (Study No. AJIRB-BMR-KSP-19-059).

\section{RNA extraction and gene expression analysis}

Total RNA was isolated from fresh frozen surgical tissues using the RNeasy mini kit (Qiagen, Hilden, Germany). Realtime quantitative polymerase chain reaction (RT-qPCR) was performed using the SensiFAST ${ }^{\mathrm{TM}}$ probe Lo-ROX Kit (Bioline, London, UK). Further information is provided in Supplementary methods. The PCR primers used are described in Table S2.

\section{Statistical analysis}

Statistical analyses were performed using R, version 3.6.3, and MedCalc Statistical Software, version 19.2 (MedCalc Software Ltd, Ostend, Belgium). The recurrence free survival (RFS) was assessed using the Kaplan-Meier method. 
A threshold for statistical significance was set at $P<0.05$. Details are described in Supplementary methods.

\section{Results}

\section{Identification of a 7-gene panel for predicting recurrence and survival outcomes in diffuse type gastric cancer}

The overall workflow of this study is illustrated in Supplementary Figure S1. We undertook a systematic, comprehensive, and an unbiased biomarker discovery approach by analyzing genome-wide expression profiling data from various datasets. The GSE62254 dataset was selected for the initial biomarker discovery, which included 45 patients with recurrence and 46 without any recurrence. Initially, we identified 293 genes that were differentially expressed between tumors with vs. without recurrence $(P \leq 0.01$; absolute $\log 2$ fold change $>1)$. Thereafter, A Least Absolute Shrinkage and Selectionator Operator (LASSO) Cox regression with tenfold cross-validation and a Cox proportional hazard regression analyses allowed us to identify a panel of seven genes: HLF, CAV2, HACD1, MLF1, GC, VSNL1, and SERPINB5. As shown in Fig. 1a, among this panel, five genes were upregulated and two were downregulated in DGC with recurrence. Fig. $1 \mathrm{~b}$ illustrates a heatmap for the cluster analysis for the seven-gene expression in relationship with several clinical features including patient age, gender, tumor stage, and tumor recurrence.

Next, we developed a logistic regression model to predict tumor recurrence in patients with DGC, which yielded an area under curve (AUC) value of 0.91 (95\% CI 0.83-0.96, $P<0.001$ ). The performance of the 7-gene panel for its ability to discriminate recurrence group from non-recurrence group is depicted in the waterfall plot in Fig. 1c. Thereafter, the predictive accuracy of this gene panel was validated in two independent datasets. In the validation cohort 1 (GSE13861), the performance of our biomarker was remarkable as evidenced by the AUC value of 0.86 (95\% CI 0.66-0.96, $P<0.001$; Supplementary Figure S2A), which was subsequently confirmed again in the validation cohort 2
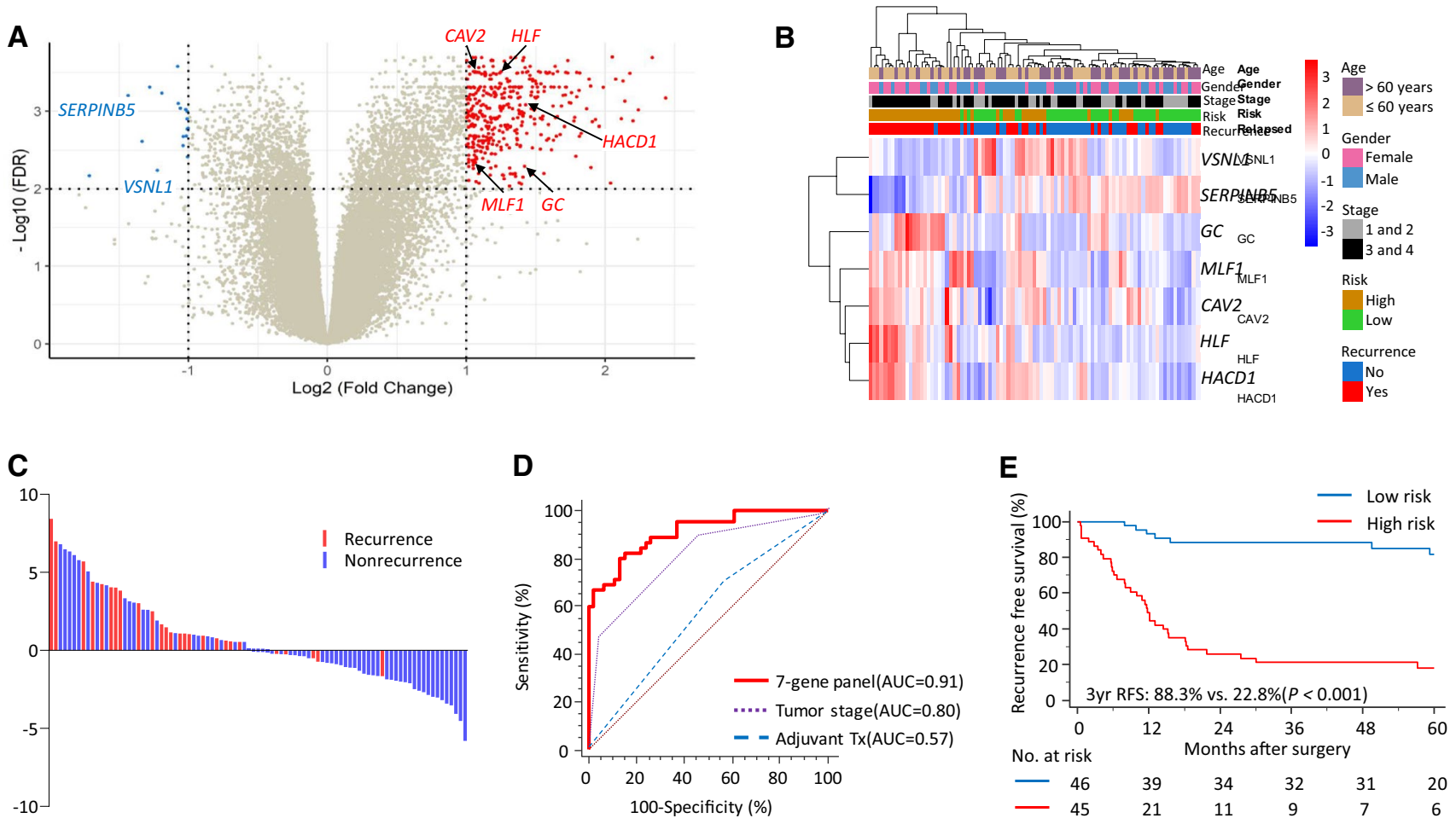

Fig. 1 Discovery of a 7-gene panel and its performance to predict recurrence in a genome-wide expression profiling dataset (GSE62254). a A volcano plot illustrating 7 genes discovered from several bioinformatic approaches. Among 293 transcriptomes that were differentially expressed in tumors with and without recurrence, a LASSO regression and a Cox proportional hazard regression analysis reduced this to a 7-gene panel. b A heatmap showing clustering of gene expression based on clinical variables as well as tumor recur- rence information. c A waterfall plot demonstrating the performance of the 7-gene panel to discriminate cases with recurrence from those without relapse. d A ROC curve depicting the recurrence-prediction accuracy was significantly superior to the other clinical factors such as tumor stage and adjuvant chemotherapy. e A Kaplan-Meier curve illustrating the significant survival differences between the two risk groups derived from the biomarker panel 
(TCGA; AUC $=0.70,95 \%$ CI 0.54-0.84, $P<0.001$; Supplementary Figure S2B). Taken together, our biomarker discovery effort utilizing rigorous bioinformatic modules allowed us to successfully identify and validate a 7-gene panel that performed robustly in predicting recurrence in independent datasets of DGC patients.

In order to further evaluate the clinical significance of our recurrence prediction biomarkers, we next investigated the prognostic implication of this 7-gene panel. In a multivariate Cox proportional hazard analysis, we noted that our 7-gene panel was the most significant prognostic indicator for RFS in all cohorts (Table S3). Thereafter, we compared the performance of the gene panel in the discovery cohort with various clinical factors using logistic regression analysis, which demonstrated that the predictive accuracy of the gene panel was remarkably higher than either the tumor stage or the adjuvant treatment (Fig. 1d). Likewise, when we evaluated the predictive accuracy of these biomarkers in the validation cohorts, our findings were yet again confirmed and the performance of the gene panel to predict recurrence was significantly higher compared to other clinical variables (Supplementary Figure S2A and B). Considering that the pathologic tumor stage is an established prognostic indicator in GC, we next combined our 7-gene panel with stage information and assessed the predictive power of this combination panel together. Intriguingly, this combination led to further improvement in the recurrence prediction potential of our gene panel and yielded a significantly higher AUC value of 0.97 in the discovery cohort (vs. the AUC of 0.91 with the gene panel alone) and 0.89 in validation cohort 1 (vs. an AUC of 0.86 with the gene panel), respectively (Table 2).

Next, we analyzed the prognostic potential of our gene panel in various patient cohorts. The Kaplan-Meier analysis in the discovery cohort revealed that based upon our recurrence prediction model, patients categorized within the high-risk group had a significantly worse survival vs. those in the low-risk group (3-year RFS: $88.3 \%$ vs. $22.8 \%$, $P<0.001$; Fig. 1e). This significant survival difference was also observed in the validation cohort 1 (3-year RFS: $84.6 \%$ vs. $58.7 \%, P=0.029$; Supplementary Figure S2C) and the validation cohort 2 (3-year RFS: $80.0 \%$ vs. $48.6 \%, P=0.041$; Supplementary Figure S2D). Taken together, these results highlight that our 7-gene panel is quite robust in predicting relapse in patients with DGC.

\section{Training and establishment of a risk-stratification model for recurrence prediction in patients with DGC}

Following successful discovery and validation of our gene panel, we next undertook an effort to further refine our biomarker panel and establish a risk-prediction model by training our biomarkers in a clinical training cohort of 180 DGC patients, by performing RT-qPCR assays for each of the 7 genes. We developed a 7 -gene panel using a single-step logistic regression analysis, which exhibited an AUC value of 0.78 (95\% CI $0.71-0.84$, sensitivity $87.2 \%$, specificity $62.7 \%, P<0.001$; Table 2). The performance of this gene panel for recurrence prediction was assessed in other types of histology (intestinal and mixed type) with tissue samples from $60 \mathrm{GC}$ patients, but it was not significant (data not shown).

In the training cohort, Cox proportional hazard analysis demonstrated that our gene panel, tumor stage, and the presence of lymphovascular invasion (LVI) by tumor cells were significant prognostic indicators in the univariate analysis (Fig. 2a). Among these, tumor stage (HR: 3.24, 95\% CI 1.87-5.61, $P<0.001$ ) and the 7-gene panel (HR: 2.23, 95\% CI 1.37-3.64, $P=0.001$ ), representing the patients within the high-risk group, were associated with a significantly
Table 2 Summary of the biomarker performance for its ability to predict recurrence in patients with diffuse type gastric cancer

\begin{tabular}{llllll}
\hline Cohorts & AUC & $95 \%$ CI & Accuracy & Sensitivity & Specificity \\
\hline $\begin{array}{l}\text { Discovery cohort (GSE62254) } \\
\text { Gene panel }\end{array}$ & 0.91 & $0.83-0.96$ & 0.82 & 0.82 & 0.85 \\
$\quad$ Gene panel and tumor stage & 0.97 & $0.90-0.99$ & 0.91 & 0.89 & 0.93 \\
Validation cohort (GSE13861) & & & & & \\
$\quad$ Gene panel & 0.86 & $0.66-0.96$ & 0.85 & 0.72 & 1.00 \\
$\quad$ Gene panel and tumor stage & 0.89 & $0.70-0.98$ & 0.81 & 0.82 & 0.87 \\
Analysis with clinical samples & & & & & \\
Clinical training cohort & & & & & 0.62 \\
$\quad$ Gene panel & 0.78 & $0.71-0.84$ & 0.71 & 0.87 & 0.78 \\
$\quad$ Gene panel and tumor stage & 0.83 & $0.76-0.88$ & 0.76 & 0.79 & 0.70 \\
Clinical validation cohort & & & & & 0.94 \\
$\quad$ Gene panel & 0.83 & $0.72-0.90$ & 0.77 & 0.84 \\
$\quad$ Gene panel and tumor stage & 0.89 & $0.80-0.95$ & 0.86 & 0.84 & \\
\hline
\end{tabular}

$A U C$ area under curve, $C I$ confidence interval 
A

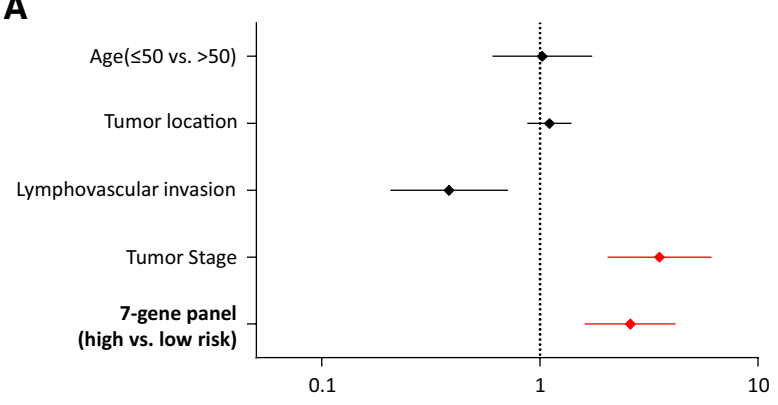

C

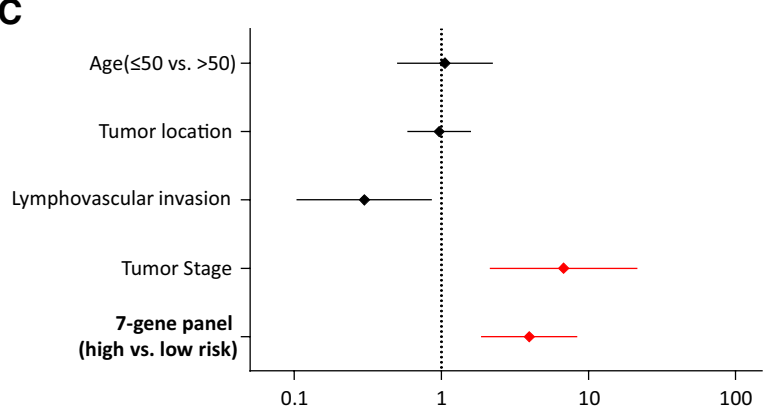

B

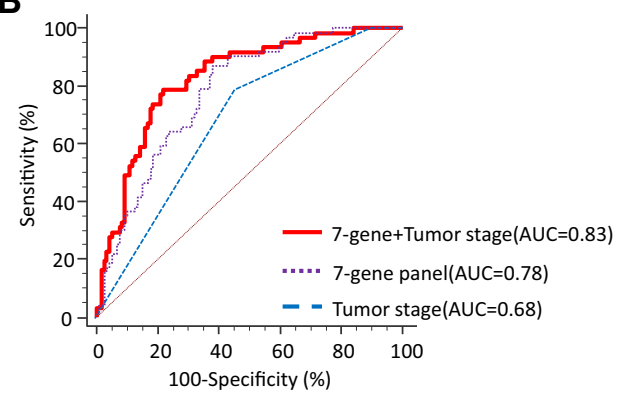

D

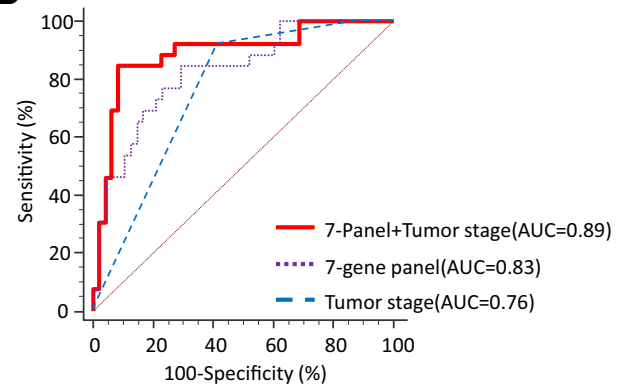

Fig. 2 Prognostic significance and performance of the biomarker panel to predict recurrence in clinical cohorts. In the training cohort, a a multivariate Cox proportional hazard analysis indicated that the 7-gene biomarker panel and tumor stage were significant factors for predicting recurrence free survival. b A ROC curve showed the enhanced predictive accuracy for recurrence of the biomarker panel

shorter RFS in the multivariate analysis (Table S4). In view of these findings, we next questioned whether a combination of our gene panel along with pathologic tumor stage might further improve the performance of the recurrence prediction model. Indeed, when we conducted these analysis, we noted that the combination of the gene panel together with the tumor stage further improved the predictive accuracy (AUC $=0.83$; 95\% CI 0.76-0.88, sensitivity: $79.2 \%$, specificity: $78.3 \%, P<0.001$ vs. AUC of 0.78 for the gene panel alone; Fig. 2b).

Through these analyses, we yet again confirmed that the integration of our gene biomarker together with stage information led to a significantly improved performance in predicting recurrence in patients with DGC.

\section{The risk-prediction model successfully identified high-risk patients with recurrence in an independent validation cohort}

Following the development of the risk prediction model, we evaluated its performance in an independent cohort of 74 patients. It was quite encouraging to observe that our biomarker panel successfully discriminated patients who experienced recurrence from those without relapse, with a along with the tumor stage. In the validation cohort, $\mathbf{c}$ the multivariate analysis consistently demonstrated that gene biomarker panel and tumor stage were significant prognostic indicators. $\mathbf{d}$ The ROC curve also exhibited the augmented performance of the biomarker panel in its ability to predict tumor recurrence when combined with tumor stage information

corresponding AUC value of 0.83 (95\% CI $0.72-0.90$, sensitivity: $77.8 \%$, specificity: $84.8 \%, P=0.006$; Table 2 ). In line with our training phase data, a univariate Cox analysis in the validation cohort also demonstrated that tumor stage and our gene panel along with the presence of LVI were significantly associated with poor prognosis (Fig. 2c). A multivariate analysis again demonstrated that tumor stage (HR 4.60, 95\% CI 1.39-15.17, $P=0.012)$ and the 7-gene panel (HR 2.71, 95\% CI 1.25-5.86, $P=0.012$ ) were significant prognostic factors in DGC patients (Table S4). Likewise, the combination of the tumor stage further augmented the predictive accuracy of the gene panel (AUC $=0.89,95 \% \mathrm{CI}$ $0.80-0.95$, sensitivity: $84.7 \%$, specificity $92.3 \%, P<0.001$ vs. AUC of 0.83 for the gene panel; Fig. 2d). Collectively, this independent validation of the recurrence prediction model confirmed the robustness of our biomarker panel for its ability to predict recurrence in patients with DGC.

\section{The 7-gene panel robustly predicted prognosis of patients with DGC}

Considering that tumor recurrence is almost always accompanied with poor survival in GC patients, we next evaluated the prognostic potential of our biomarker panel in 
the clinical cohort of patients with DGC. Patients were dichotomized into high- and low-risk groups for recurrence according to the cutoff threshold values derived from the prediction model, followed by Kaplan-Meier analyses to determine the prognostic significance of our gene panel. In the training cohort, RFS in the high-risk group patients was significantly worse compared to those in the low-risk group (3-year RFS: $73.4 \%$ vs. $43.7 \%, P<0.001$; dotted lines in Fig. 3a). Next, we assessed the potential of the prognostic classifier consisting of the gene panel and tumor stage, which further enhanced the prognostic accuracy for defining survival differences (3-year RFS: $75.3 \%$ vs. $37.5 \%$, $P<0.001$; solid lines in Fig. 3a). Similarly, in the validation cohort, we were able to successfully validate these findings, wherein our gene biomarker panel robustly dichotomized patients into low- and high-risk groups (3-year RFS: 74.5\% vs. $35.3 \%, P<0.001$; dotted lines in Fig. $3 b$ ), and this prognostic accuracy was further enhanced when the gene panel
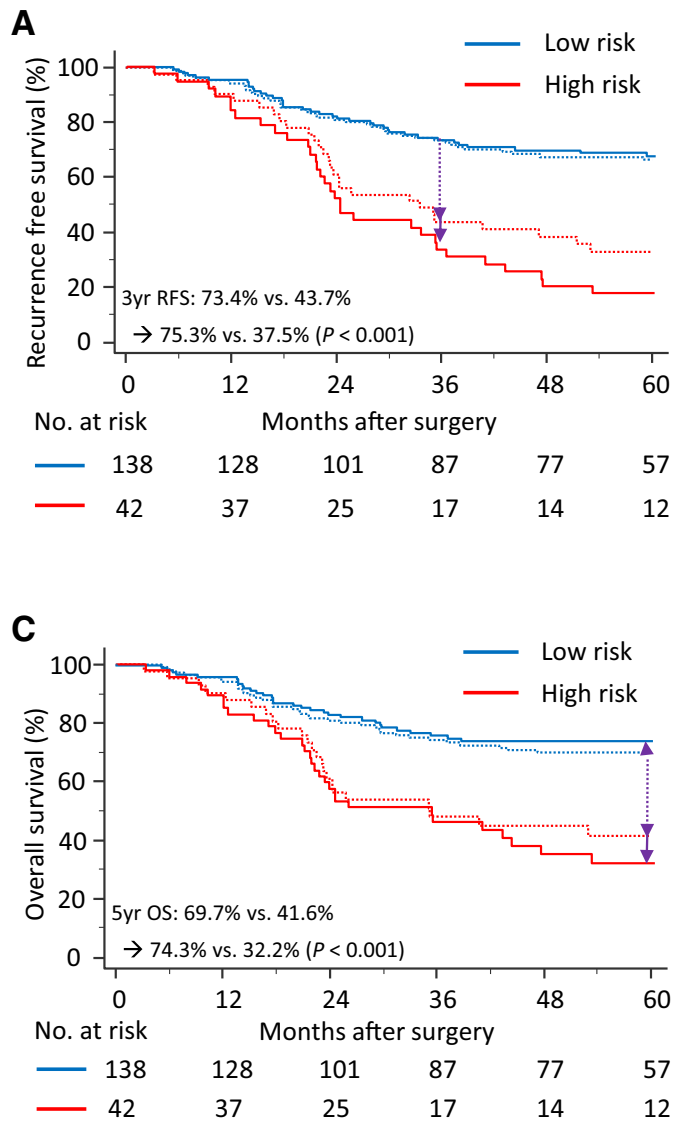

Fig. 3 Prognostic implication of the 7-gene biomarker panel for predicting patient survival as determined by the Kaplan-Meier analyses. Kaplan-Meier curves exhibited the significant differences in recurrence free survival between two risk groups determined by the cutoff values derived from the 7-gene based risk prediction model (dotted lines). In addition, the enhanced ability of the 7-gene biomarker panel for its potential to determine patient prognosis when combined was combined with tumor stage (3-year RFS: $74.5 \% 23.1 \%$, $P<0.001$; solid lines in Fig. $3 b$ ).

Thereafter, we evaluated the prognostic significance of the gene panel by analyzing overall survival (OS). A significant survival difference was observed in both the training cohort (5-year OS: $69.7 \%$ vs. $41.6 \%, P=0.001$; Fig. $3 c$ ) and the validation cohort (5-year OS: $84.3 \%$ vs. $51.9 \%$, $P=0.010$; Fig. 3d). Moreover, we noted that the survival differences derived from the combination of the gene panel with tumor stage were significantly superior and exhibited a 5-year OS of $74.3 \%$ in low-risk group and $32.2 \%$ in highrisk group of the training cohort $(P<0.001$; solid lines in Fig. 3c), which was successfully validated in the independent validation cohort ( $85.5 \%$ vs. $32.4 \%, P<0.001$; solid lines in Fig. 3d).

We next examined the prognostic significance of our gene biomarker panel vs. the TNM staging system and noted that it still emerged as a better prognosticator in
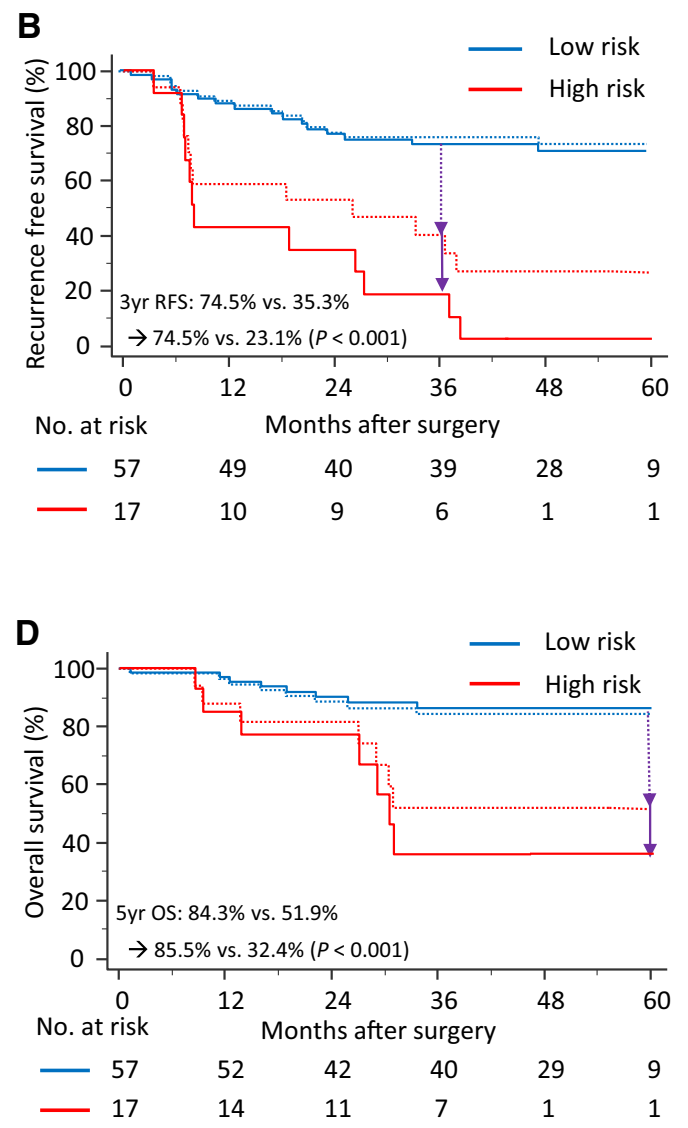

with tumor stage (solid lines) was consistently observed in a the clinical training cohort and $\mathbf{b}$ the clinical validation cohort. Likewise, in the analyses of overall survival, a high-risk patient group exhibited a significantly shorter survival compared to the low-risk group (dotted lines) and the prognostic differences were augmented when tumor stage was integrated (solid lines) in $\mathbf{c}$ the training cohort and $\mathbf{d}$ the validation cohort. $R F S$ recurrence free survival, $O S$ overall survival 
stage 3 DGC patients who are frequently the victim of disease recurrence and poor survival outcomes. The gene biomarker panel successfully identified high-risk patients who exhibited unfavorable survival in the training cohort (3-year RFS: $64.9 \%$ vs. $37.0 \%, P=0.014$; Fig. 4 a), as well as the validation cohort patients (3-year RFS: $60.8 \%$ vs. $32.0 \%, P=0.013$; Fig. 4 b). We also ascertained that our gene biomarkers stratified the prognosis in patients with stage 2 DGC (3-year RFS of the training cohort: $89.1 \%$ vs. $70.0 \%, P=0.025$ and the validation cohort: $95.0 \%$ vs. $50.0 \%, P=0.019$; Supplementary Figure S3). These results highlight that, in addition for its ability to predict disease relapse, our gene biomarker is robust and clinically significant in stratifying the prognosis of patients with DGC.

\section{Establishment of a nomogram for an easier clinical translation for predicting prognosis in patients with DGC}

In view of the translational and clinical focus of our study, we established a nomogram comprising of the 7-gene panel and the tumor stage, which robustly exhibited a significance in multiple cohorts for predicting RFS. Each parameter was assigned a score according to its probability for predicting recurrence, and the sum of the scores was used to predict 3 and 5-year RFS. This nomogram ascertained that the largest contributions to predict the RFS was the 7-gene biomarker panel (Fig. 4c), emphasizing the clinical significance of our biomarker panel for an easier clinical translation for predicting prognostic outcomes in patients with DGC.
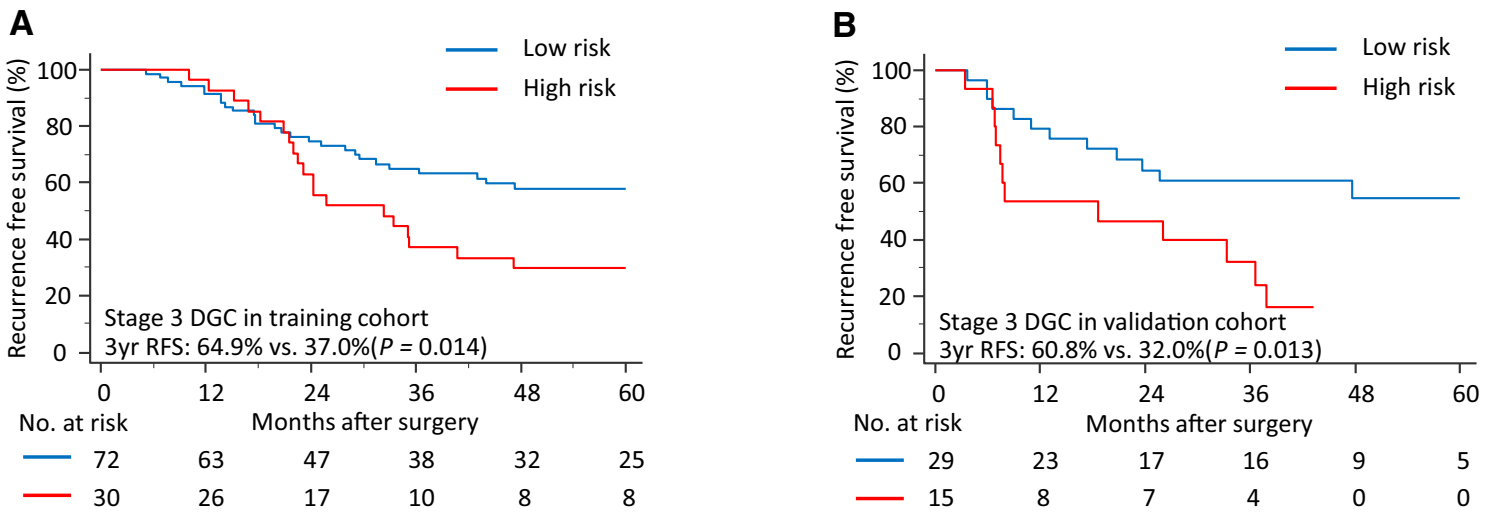

C

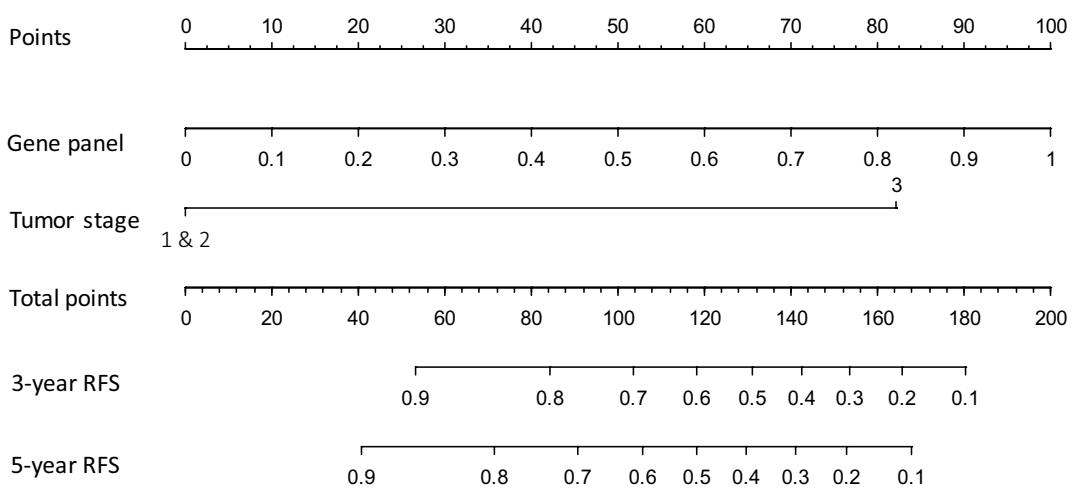

Fig. 4 Clinical significance of the 7-gene biomarker panel in patients with stage 3 diffuse type gastric cancer and a nomogram to assess patients' survival in clinical cohorts. The gene biomarker panel retained its prognostic significance independent of the tumor stage and successfully identified high-risk patients with stage 3 disease who frequently experience relapse and exhibit poor outcomes. A sig- nificantly unfavorable prognosis was observed in high-risk patients within $\mathbf{a}$ the training cohort and $\mathbf{b}$ the validation cohort. A nomogram to evaluate the recurrence free survival was established and it ascertained the major contribution of the 7-gene biomarker panel for the assessment of prognosis in patients with DGC. RFS recurrence free survival 


\section{Discussion}

Poor response to conventional chemotherapy and heterogenous disease outcomes makes the clinical decision-making for selecting appropriate treatment modalities and predicting survival outcomes in patients with DGC challenging. This highlights the need for the development of robust risk-stratification models for performing the risk assessment and identification of subset of patient groups at higher risk for recurrence and those who might benefit from newer treatment strategies. With the advances in high-throughput genomic sequencing technologies, there has been a resurgence of molecular biomarker development that can complement the currently used pathological prognostic indicators in the clinical settings. For instance, in breast and colorectal cancer, such molecular biomarkers are now routinely being used clinically to stratify patient subgroups for determining prognostic outcomes and predicting chemotherapeutic responses [19-21]. However, in spite of ongoing efforts at unveiling the genomic and epigenomic landscape in GC, much of these molecular insights have had a very little impact on the clinical management of patients suffering from this malignancy. In particular, there is a clear lack of availability of molecular biomarkers that can stratify patient subsets with frequent recurrence and poor prognosis - which has important implications for guiding optimal treatment strategies and improving overall disease outcomes.

A recent proteomic analysis observed that DGC exhibits significant tumor heterogeneity and can be classified into three distinctive subtypes that associate with very distinct clinical outcomes [22]. This study demonstrated that patients with tumor subtypes exhibiting dysregulation in the cell cycle or EMT process revealed a favorable response to chemotherapy, while the other subtype was enriched in immune response proteins and were insensitive to chemotherapy, leading to the worse prognosis. More intriguingly, this study indicated that expression of several druggable proteins was highly enriched in this last subset of tumors with dismal prognosis and suggested that these might benefit from targeted immunotherapy. The findings from our study were relevant in this regard as our results also supported that patients with DGC comprise of subsets of patient group with different clinical outcomes depending on the response to various treatment. Our study is particularly of interest, as our biomarker panel allowed riskstratification and identification of high-risk patients who are vulnerable for disease recurrence, as these patients are the ones who could be offered such novel therapeutic modalities since they very unlikely will not respond to currently used conventional adjuvant chemotherapeutic drugs.

From a biological and functional viewpoint, majority of the genes in our biomarker panel have previously been reported to play an important role in cancer pathogenesis. For instance, Caveolin-2 (CAV2) along with $C A V 1$, which plays a pivotal role in signal transduction and gene regulation of cell growth/apoptosis, have been shown to involve in cancer initiation and metastasis [23, 24]. Myeloid leukemia factor $1(M L F 1)$ is a gene that encodes a protein that functions in the phenotypic determination of hematopoietic cells. This gene is known to be frequently methylated in GC and is characterized by different levels of methylation according to various stages during tumor progression [25]. Likewise, EMT is a key step for cancer cells to metastasize [26]. Serpin family B member 5 (SER$P I N B 5)$ participates in the integrated pathway of transforming growth factor $\beta$ (TGF- $\beta$ )/Snail with TNF $\alpha / \mathrm{NF \kappa B}$ which drives EMT and is associated with GC progression [27, 28]. Visinin-like protein-1 (VSNL1) acts as a tumor suppressor gene and it is reported to be associated with lymph node metastasis and the prognosis in colorectal cancer [29, 30]. $G C$ gene encoding the vitamin D-binding protein is highly polymorphic and its polymorphism is associated with pathogenesis of colon and breast cancer [31, 32]. Hepatic leukemia factor (HLF) plays an important role in apoptosis of fibroblasts and aberrant expression of the gene is correlated with early progression and distant metastasis in non-small cell lung cancer as well as hematological malignancy and glioma [33-35].

We would like to acknowledge some of the potential limitations of our study. First, in spite of the analysis of multiple independent patient cohorts in this study, ours was a retrospective study. Second, as per the current treatment guidelines, the majority of patients with stage 2 and 3 DGC in clinical cohorts received adjuvant chemotherapy, we were unable to evaluate the potential of our biomarker panel to predict benefits from conventional chemotherapy. To overcome these limitations, well-designed and multi-institutional prospective studies must validate our results prior to their translation into the clinic.

In conclusion, through a systematic and comprehensive discovery and validation effort, we have developed a novel gene expression biomarker panel that can predict recurrence in patients with diffuse type gastric cancer. We established a risk-stratification model for the identification of high-risk patients with adverse outcomes and prediction of prognosis-which could potentially facilitate individualized treatment and improving the survival outcomes in patients suffering from this malignancy.

Acknowledgements We would like to thank Yuma Wada for developing a nomogram and Yasuyuki Okada for helping with their important insights into experiments and data analysis. The biospecimens and data used in this study were provided by Asan Bio-Resource Center, Korea Biobank Network 2019-05(184) and the Ajou Human Bio-Resource 
Bank (AHBB), a member of the National Biobank of Korea, which is supported by the Ministry of Health and Welfare. The present work was supported by the Grants CA72851, CA181572, and CA187956 from the National Cancer Institute, National Institutes of Health, and a pilot grant from the Stupid Strong Foundation to A. Goel.

Author contributions IL contributed to study concept and design; specimen provider; acquisition of data; analysis and interpretation of data; statistical analysis; drafting of the manuscript. DS contributed to analysis and interpretation of data; statistical analysis; drafting of the manuscript. HH contributed to specimen provider; acquisition of data; drafting of the manuscript. JY contributed to specimen provider; acquisition of data; critical revision of the manuscript. BK contributed to specimen provider; acquisition of data; critical revision of the manuscript. AG contributed to study concept and design; analysis and interpretation of data; statistical analysis; drafting of the manuscript; critical revision of the manuscript.

Funding The present work was supported by the Grants CA72851, CA181572, and CA187956 from the National Cancer Institute, National Institutes of Health, and a pilot grant from the Stupid Strong Foundation to A. Goel.

\section{Compliance with ethical standards}

Conflict of interest None of the authors has any potential conflicts to disclose.

Human rights statement and informed consent All procedures followed were in accordance with Institutional Review Board of Asan Medical Center (Study No. 2019-0237) and Ajou university hospital (Study No. AJIRB-BMR-KSP-19-059), and with the Helsinki Declaration of 1964 and later versions. Informed consent was obtained from all patients.

\section{References}

1. Ferlay J, Colombet M, Soerjomataram I, Mathers C, Parkin DM, Pineros $\mathrm{M}$, et al. Estimating the global cancer incidence and mortality in 2018: GLOBOCAN sources and methods. Int J Cancer. 2019;144(8):1941-53. https://doi.org/10.1002/ijc.31937 (Epub 2018/10/24 PubMed PMID: 30350310).

2. Bang YJ, Kim YW, Yang HK, Chung HC, Park YK, Lee KH, et al. Adjuvant capecitabine and oxaliplatin for gastric cancer after D2 gastrectomy (CLASSIC): a phase 3 open-label, randomised controlled trial. Lancet. 2012;379(9813):315-21. https://doi. org/10.1016/S0140-6736(11)61873-4 (Epub2012/01/10PubMe dPMID:22226517)

3. Sakuramoto S, Sasako M, Yamaguchi T, Kinoshita T, Fujii M, Nashimoto A, et al. Adjuvant chemotherapy for gastric cancer with S-1, an oral fluoropyrimidine. N Engl J Med. 2007;357(18):1810 20. https://doi.org/10.1056/NEJMoa072252 (Epub 2007/11/06 PubMed PMID: 17978289).

4. Cunningham D, Allum WH, Stenning SP, Thompson JN, Van de Velde CJ, Nicolson M, et al. Perioperative chemotherapy versus surgery alone for resectable gastroesophageal cancer. N Engl J Med. 2006;355(1):11-20. https://doi.org/10.1056/NEJMoa0555 31 (Epub 2006/07/11 PubMed PMID: 16822992).

5. Cheong JH, Yang HK, Kim H, Kim WH, Kim YW, Kook $\mathrm{MC}$, et al. Predictive test for chemotherapy response in resectable gastric cancer: a multi-cohort, retrospective analysis. Lancet Oncol. 2018;19(5):629-38. https://doi.org/10.1016/
S1470-2045(18)30108-6 (Epub 2018/03/24 PubMed PMID: 29567071).

6. Oh SC, Sohn BH, Cheong JH, Kim SB, Lee JE, Park KC, et al. Clinical and genomic landscape of gastric cancer with a mesenchymal phenotype. Nat Commun. 2018;9(1):1777. https ://doi.org/10.1038/s41467-018-04179-8 (Epub 2018/05/05 PubMed PMID: 29725014; PubMed Central PMCID: PMCPMC5934392).

7. Lauren P. The two histological main types of gastric carcinoma: diffuse and so-called intestinal-type carcinoma. An attempt at a histo-clinical classification. Acta Pathol Microbiol Scand. 1965;64:31-49. https://doi.org/10.1111/apm.1965.64.1.31 (Epub 1965/01/01 PubMed PMID: 14320675)

8. Lordick F, Janjigian YY. Clinical impact of tumour biology in the management of gastroesophageal cancer. Nat Rev Clin Oncol. 2016;13(6):348-60. https://doi.org/10.1038/nrclinonc.2016.15 (Epub 2016/03/02. PubMed PMID: 26925958; PubMed Central PMCID: PMCPMC5521012).

9. Cancer Genome Atlas Research N. Comprehensive molecular characterization of gastric adenocarcinoma. Nature. 2014;513(7517):202-9. https://doi.org/10.1038/natur e13480 (Epub 2014/08/01. PubMed Central PMCID: PMCPMC4170219).

10. Cristescu R, Lee J, Nebozhyn M, Kim KM, Ting JC, Wong SS, et al. Molecular analysis of gastric cancer identifies subtypes associated with distinct clinical outcomes. Nat Med. 2015;21(5):449_ 56. https://doi.org/10.1038/nm.3850 (Epub 2015/04/22. PubMed PMID: 25894828).

11. Sohn BH, Hwang JE, Jang HJ, Lee HS, Oh SC, Shim JJ, et al. Clinical significance of four molecular subtypes of gastric cancer identified by the cancer genome atlas project. Clin Cancer Res. 2017. https://doi.org/10.1158/1078-0432.CCR-16-2211 (Epub 2017/07/28. PubMed PMID: 28747339; PubMed Central PMCID: PMCPMC5785562).

12. Wong SS, Kim KM, Ting JC, Yu K, Fu J, Liu S, et al. Genomic landscape and genetic heterogeneity in gastric adenocarcinoma revealed by whole-genome sequencing. Nat Commun. 2014;5:5477. https://doi.org/10.1038/ncomms6477 (Epub 2014/11/20. PubMed PMID: 25407104).

13. Jeon TY, Han ME, Lee YW, Lee YS, Kim GH, Song GA, et al. Overexpression of stathmin 1 in the diffuse type of gastric cancer and its roles in proliferation and migration of gastric cancer cells. Br J Cancer. 2010;102(4):710-8. https://doi.org/10.1038/ sj.bjc.6605537 (Epub 2010/01/21. PubMed PMID: 20087351; PubMed Central PMCID: PMCPMC2837578).

14. Ueda T, Volinia S, Okumura H, Shimizu M, Taccioli C, Rossi S, et al. Relation between microRNA expression and progression and prognosis of gastric cancer: a microRNA expression analysis. Lancet Oncol. 2010;11(2):136-46. https://doi.org/10.1016/ S1470-2045(09)70343-2 (Epub 2009/12/22. PubMed PMID: 20022810; PubMed Central PMCID: PMCPMC4299826).

15. McShane LM, Altman DG, Sauerbrei W, Taube SE, Gion M, Clark GM, et al. Reporting recommendations for tumor marker prognostic studies (REMARK). J Natl Cancer Inst. 2005;97(16):1180-4. https://doi.org/10.1093/jnci/dji237 (Epub 2005/08/18. PubMed PMID: 16106022)

16. Flejou JF. WHO Classification of digestive tumors: the fourth edition. Ann Pathol. 2011;31(5 Suppl):S27-31. https://doi. org/10.1016/j.annpat.2011.08.001 (Epub 2011/11/30. PubMed PMID: 22054452).

17. Japanese Gastric Cancer A. Japanese gastric cancer treatment guidelines 2014 (ver. 4). Gastric Cancer. 2017;20(1):1-19. https://doi.org/10.1007/s10120-016-0622-4 (Epub 2016/06/28. PubMed PMID: 27342689; PubMed Central PMCID: PMCPMC5215069 Phamaceutical, Sanofi, Merck Serono, Yakult Honsha, Daiichi Sankyo, Otsuka Pharmaceutical 
Factory, Takeda Pharmaceutical, Johnson \& Johnson, Asahi Kasei Pharma, Eli Lilly Japan, Pfizer Japan, AJINOMOTO Pharmaceuticals, ONO Pharmaceutical and Kaken Pharmaceutical and grants from Covidien Japan, Shionogi, Bristol Myers Squib, Japan Blood Products Organization, Torii Pharmaceutical, Mitsubishi Tanabe Pharma, bbVie GK, Otsuka Pharmaceutical, Yoshindo, Eizai, Abbott Japan, CSL Behring, Teijin Pharma, Tsumura, Nippon Kayaku, Miyarisan Pharmaceutical, Novartis Pharmaceuticals Japan, KCI, Toyama Chemical, Maruho, Hogy Medical and MSD, outside the submitted work. Dr. Sano reports personal fees from Chugai Phamaceutical, Covidien Japan, Eli Lilly Japan, Johnson \& Johnson, Olympus, Otsuka Pharmaceutical Factory, Taiho Pharmaceutical and Yakult Honsha).

18. Edge SB, Compton CC. The American Joint Committee on Cancer: the 7th edition of the AJCC cancer staging manual and the future of TNM. Ann Surg Oncol. 2010;17(6):1471-4. https://doi. org/10.1245/s10434-010-0985-4 (Epub 2010/02/25. PubMed PMID: 20180029).

19. Paik S, Shak S, Tang G, Kim C, Baker J, Cronin M, et al. A multigene assay to predict recurrence of tamoxifen-treated, node-negative breast cancer. N Engl J Med. 2004;351(27):2817-26. https ://doi.org/10.1056/NEJMoa041588 (Epub 2004/12/14. PubMed PMID: 15591335).

20. Paik S, Tang G, Shak S, Kim C, Baker J, Kim W, et al. Gene expression and benefit of chemotherapy in women with nodenegative, estrogen receptor-positive breast cancer. J Clin Oncol. 2006;24(23):3726-34. https://doi.org/10.1200/JCO.2005.04.7985 (Epub 2006/05/25. PubMed PMID: 16720680).

21. Song N, Pogue-Geile KL, Gavin PG, Yothers G, Kim SR, Johnson $\mathrm{NL}$, et al. Clinical outcome from oxaliplatin treatment in stage II/ III colon cancer according to intrinsic subtypes: secondary analysis of NSABP C-07/NRG oncology randomized clinical trial. JAMA Oncol. 2016;2(9):1162-9. https://doi.org/10.1001/jamao ncol.2016.2314 (Epub 2016/06/09. PubMed PMID: 27270348; PubMed Central PMCID: PMCPMC5065181).

22. Ge S, Xia X, Ding C, Zhen B, Zhou Q, Feng J, et al. A proteomic landscape of diffuse-type gastric cancer. Nat Commun. 2018;9(1):1012. https://doi.org/10.1038/s41467-018-03121-2 (Epub 2018/03/10. PubMed PMID: 29520031; PubMed Central PMCID: PMCPMC5843664).

23. Chen D, Che G. Value of caveolin-1 in cancer progression and prognosis: Emphasis on cancer-associated fibroblasts, human cancer cells and mechanism of caveolin-1 expression (Review). Oncol Lett. 2014;8(4):1409-21. https://doi.org/10.3892/ol.2014.2385 (Epub 2014/09/10. PubMed PMID: 25202343; PubMed Central PMCID: PMCPMC4156192).

24. Mercier I, Jasmin JF, Pavlides S, Minetti C, Flomenberg N, Pestell $\mathrm{RG}$, et al. Clinical and translational implications of the caveolin gene family: lessons from mouse models and human genetic disorders. Lab Invest. 2009;89(6):614-23. https://doi.org/10.1038/labin vest.2009.23 (Epub 2009/04/01. PubMed PMID: 19333235; PubMed Central PMCID: PMCPMC2796209).

25. Watanabe Y, Kim HS, Castoro RJ, Chung W, Estecio MR, Kondo $\mathrm{K}$, et al. Sensitive and specific detection of early gastric cancer with DNA methylation analysis of gastric washes. Gastroenterology. 2009;136(7):2149-58. https://doi.org/10.1053/j.gastr o.2009.02.085 (Epub 2009/04/21. PubMed PMID: 19375421; PubMed Central PMCID: PMCPMC2722957).

26. Hugo H, Ackland ML, Blick T, Lawrence MG, Clements JA, Williams ED, et al. Epithelial-mesenchymal and mesenchymalepithelial transitions in carcinoma progression. J Cell Physiol.
2007;213(2):374-83. https://doi.org/10.1002/jcp.21223 (Epub 2007/08/08. PubMed PMID: 17680632).

27. Lei KF, Liu BY, Zhang XQ, Jin XL, Guo Y, Ye M, et al. Development of a survival prediction model for gastric cancer using serine proteases and their inhibitors. Exp Ther Med. 2012;3(1):109-16. https://doi.org/10.3892/etm.2011.353 (Epub 2012/09/13. PubMed PMID: 22969854; PubMed Central PMCID: PMCPMC3438548).

28. Li H, Zhong A, Li S, Meng X, Wang X, Xu F, et al. The integrated pathway of TGFbeta/Snail with TNFalpha/NFkappaB may facilitate the tumor-stroma interaction in the EMT process and colorectal cancer prognosis. Sci Rep. 2017;7(1):4915. https ://doi.org/10.1038/s41598-017-05280-6 (Epub 2017/07/09. PubMed PMID: 28687755; PubMed Central PMCID: PMCPMC5501852).

29. Pitule P, Vycital O, Bruha J, Novak P, Hosek P, Treska V, et al. Differential expression and prognostic role of selected genes in colorectal cancer patients. Anticancer Res. 2013;33(11):4855-65 (Epub 2013/11/14 PubMed PMID: 24222123).

30. Akagi T, Hijiya N, Inomata M, Shiraishi N, Moriyama M, Kitano $\mathrm{S}$. Visinin-like protein-1 overexpression is an indicator of lymph node metastasis and poor prognosis in colorectal cancer patients. Int J Cancer. 2012;131(6):1307-17. https://doi.org/10.1002/ ijc. 27341 (Epub 2011/11/05 PubMed PMID: 22052372).

31. Zhu Y, Wang PP, Zhai G, Bapat B, Savas S, Woodrow JR, et al. Association of rs2282679 A $>$ C polymorphism in vitamin D binding protein gene with colorectal cancer risk and survival: effect modification by dietary vitamin D intake. BMC Cancer. 2018;18(1):155. https://doi.org/10.1186/s12885-018-4026-1 (Epub 2018/02/08 PubMed PMID: 29409465; PubMed PMID: 29409465).

32. Abbas S, Linseisen J, Slanger T, Kropp S, Mutschelknauss EJ, Flesch-Janys D, et al. The Gc2 allele of the vitamin D binding protein is associated with a decreased postmenopausal breast cancer risk, independent of the vitamin D status. Cancer Epidemiol Biomarkers Prev. 2008;17(6):1339-43. https://doi. org/10.1158/1055-9965.EPI-08-0162 (Epub 2008/06/19 PubMed PMID: 18559548)

33. Chen J, Liu A, Lin Z, Wang B, Chai X, Chen S, et al. Downregulation of the circadian rhythm regulator HLF promotes multipleorgan distant metastases in non-small cell lung cancer through PPAR/NF-kappab signaling. Cancer Lett. 2020;482:56-71. https ://doi.org/10.1016/j.canlet.2020.04.007 (Epub 2020/04/15 PubMed PMID: 32289442).

34. Wahlestedt M, Ladopoulos V, Hidalgo I, Sanchez Castillo M, Hannah R, Sawen P, et al. Critical modulation of hematopoietic lineage fate by hepatic leukemia factor. Cell Rep. 2017;21(8):2251-63. https://doi.org/10.1016/j.celrep.2017.10.112 (Epub 2017/11/23 PubMed PMID: 29166614; PubMed Central PMCID: PMCPMC5714592).

35. Chen S, Wang Y, Ni C, Meng G, Sheng X. HLF/miR-132/TTK axis regulates cell proliferation, metastasis and radiosensitivity of glioma cells. Biomed Pharmacother. 2016;83:898-904. https ://doi.org/10.1016/j.biopha.2016.08.004 (Epub 2016/10/25 PubMed PMID: 27522003).

Publisher's Note Springer Nature remains neutral with regard to jurisdictional claims in published maps and institutional affiliations. 